transfer of learning possibly through setting new functional connections between cortex and the cerebellum. Although the study by Olsson et al. (2008) is limited to the training of an elementary motor act, finger tapping, the combined training effect of physical and mental training, and the transfer effect could have important consequences for training protocols for athletes in need of improved performance in, e.g., jumping or running, and for stroke patients participating in rehabilitation training programs.

\section{REFERENCES}

Ersland, L., Rosen, G., Lundervold, A., Smievoll, A. I., Tillung, T., Sundberg, H., and Hugdahl, K. (1996). Phantom limb "fingertapping" causes primary motor cortex activation: An fMRI study. Neuroreport 8 , 207-210.
Hugdahl, K., Rosen, G., Ersland, L., Lundervold, A., Smievoll, A. I., Barndon, R., and Thomsen, T. (2001). Common pathways in mental imagery and pain perception: an fMRI study of a subject with an amputated arm. Scand. J. Psychol. 42, 269-275.

Kosslyn, S. M., Ganis, G., and Thompson, W. L. (2001). Neural foundations of imagery. Nat. Rev. Neurosci. 2, 635-642.

Kosslyn, S. M., and Thompson, W.L. (2003). When is early visual cortex activated during visual mental imagery. Psychol. Bull. 129, 723-746.

Nyberg, L., Eriksson, J., Larsson, A., and Marklund, P. (2006). Learning by doing versus learning by thinking: an fMRI study of motor and mental training. Neuropsychologia 44, 711-717.

Olsson, C.-J., Jonsson, B., and Nyberg, L. (2008). Learning by doing and learning by thinking: an fMRI study combining motor and mental training. Front. Hum. Neurosci. 1. doi: 10.3389/neuro.09.005.2008.

Rosen, G., Hugdahl, K., Ersland, L., Lundervold, A. Smievoll,A.I., Barndon, R., Sundberg,H., Thomsen, T., Roscher, B. E., Tjølsen, A., and Engelsen, B. (2001).
Different brain areas activated during imagery of painful and non-painful "finger movements" in a subject with an amputated arm. Neurocase 7, 255-260.

Setiz, J., Roland, P., Bohm, C., Greitz, T., and StoneElander, S. (1990). Motor learning in man: a positron emission tomography study. Neuroreport 1, 57-60.

Tootell, R. B. H., Hadjikhani, N. K., Mendola, J. D., Marrett, S., and Dale, A. M. (1998). From retinotopy to recognition: fMRI in human visual cortex. Trends Cogn. Sci. 2, 61-201.

Received: 17 February 2009; published: 01 May 2009 Citation: Front. Neurosci. (2009) 3,1: 5-6. doi: 10.3389/ neuro.01.008.2009

Copyright $\odot 2009$ Hugdahl. This is an open-access publication subject to an exclusive license agreement between the authors and the Frontiers Research Foundation, which permits unrestricted use, distribution, and reproduction in any medium, provided the original authors and source are credited.

\title{
The emotional consequences of being distracted
}

\section{Francisco Barceló*}

Clinical Neuropsychology Research Group, Universitat de les Illes Balears, Palma de Mallorca, Spain

*Correspondence: f.barcelo@uib.es

\section{A commentary on}

Response inhibition is linked to emotional devaluation: behavioral and electrophysiological evidence

by Monica Kiss, Jane E. Raymond, Nikki Westoby, Anna C. Nobre and Martin Eimer

Emotionally salient events have long been shown to engage attentional resources more than emotionally neutral events (Vuilleumier, 2005). In contrast, the reciprocal effect that attention also influences emotion has remained mostly unexplored in spite of everyday intuition. Imagine yourself sitting at your desk, your thoughts immersed in formulating some complex brain theory. Suddenly, the door opens and an unknown face sticks out asking: $D r$. Peters? - No, wrong office - you reply. Even if the smiling face quickly retreats in silence, it already captured your attention away, and briefly interrupted the smooth flow of your thoughts and actions. Some may find this distractor effect displeasing, particularly while handling a difficult problem, or if the interruption occurs at frequent intervals. In a research article published in Frontiers in Human Neuroscience, Kiss et al. (2008) have made an original contribution to the field by means of a novel methodology to examine the links between attentional selection and emotional valuation.

The authors tested the hypothesis that human faces would be emotionally devalued and judged as less trustworthy as a consequence of having been designated as distractors - hence requiring no overt motor response - in a previous selective attention procedure. Visual targets and distractors consisted of exemplars of human faces with distinct racial features, i.e., Caucasian versus Asian faces. Trial blocks involved two stages. Firstly, participants saw a short series of novel faces, to which they responded by pressing a button to faces of one race, and refrained from responding to faces of the other race (Figure 1). Secondly, about half-a-minute later, the same faces were judged for trustworthiness in a 4-point rating scale. Each face appeared only twice, once at response selection, and once at the affective evaluation stage, to control for familiarity effects. The response assignment of target (Go) and distractor
(Nogo) racial features was reversed halfway through the experiment.

Importantly, the neural substrates of distractor devaluation were also examined. As an index of prefrontal inhibitory control, the authors measured a negative-going brain potential peaking between 250 and $350 \mathrm{~ms}$ after the onset of Nogo distractors (often termed "Nogo N2"). The phasic negativity peaks at midfrontal scalp regions and has been considered as an electrophysiological correlate of anterior cingulate function (Nieuwenhuis et al., 2003). The intensity of Nogo N2 potentials to distractor faces was larger for faces that were rated as less trustworthy than for faces judged more positively. This indicated that the efficiency of prefrontal inhibitory control triggered by distractor faces covaried with their subsequent affective devaluation.

The phenomenon of distractor devaluation was explained from a general inhibition-based account by which the same type of inhibitory tagging responsible for top-down cognitive control could be generalized to emotional as well as "perceptual, higher cognitive, or response-related stages of processing" (Kiss et al., 2008). The 
proposal stands on evidence that distractor devaluation occurs in conditions requiring strong attentional inhibition i.e., when targets and distractors share some perceptual features (Figure 1; Fenske and Raymond, 2006). In spite of its parsimony, however, the account does not clarify why efficient response inhibition to distractors does not necessarily prevent the disruption of concurrent thoughts and actions. Instead, probabilistic stimulus-response contextual associations can explain behavioral distraction and related brain responses in terms of transient overloads in working memory capacity induced by the distractors (Barceló et al., 2008). If distractor faces induced interference and response conflict, there is possibility of transient dips being caused in cognitive control whose resolution demands prefrontal resources. From this perspective, distractor devaluation and the midfrontal negativities might relate to the rather displeasing feeling of being temporary out-of-control. This interpretation concurs with the clustering of emotional and attentional symptoms observed in some frontal lobe patients (e.g., distractibility, disinhibition and irritability).

This study paves the way to elucidate these as well as several other relevant issues on the way in which attention influences emotion: How does novelty and familiarity modulate distractor devaluation? What are the specific neural substrates and cognitive mechanisms? Can the attentional biases on emotional valuation be extended to everyday life situations such as ethnic empathy and prejudice, or sibling jealousy? Such questions will surely open up rich venues for future research.

\section{REFERENCES}

Barceló, F., and Knight, R. T. (2007). An information theoretical approach to contextual processing in the human brain: evidence from prefrontal lesions. Cereb. Cortex 17, i51-i60.

Barceló, F., Perianez, J. A., and Nyhus, E. (2008). An information theoretical approach to task-switching: evidence from cognitive brain potentials in humans. Front. Hum. Neurosci. 1, 13. doi: 10.3389/ neuro.09/013.2007.

Fenske, M. J., and Raymond, J. E. (2006). Affective influences of selective attention. Curr. Dir. Psychol. Sci. 15, 312-316.

Kiss, M., Raymond, J. E., Westoby, N., Nobre, A. C., and Eimer, M., 2008. Response inhibition is linked to emotional devaluation: behavioural and electrophysiological evidence. Front. Hum. Neurosci. 2, 13. doi: 10.3389/neuro.3309.3013.2008.

Miller, E. K. (2000). The prefrontal cortex and cognitive control. Nat. Rev. Neurosci. 1, 59-65.

Nieuwenhuis, S., Yeung, N., van den Wildenberg, W., and Ridderinkhof, K. R. (2003). Electrophysiological correlates of anterior cingulate function in a go/no-go task: effects of response conflict and trial type frequency. Cogn. Affect. Behav. Neurosci. 3, 17-26.

Vuilleumier, P. (2005). How brains beware: neural mechanisms of emotional attention. Trends Cogn. Sci. 9, 585-594.

Received: 26 February 2009; published: 01 May 2009 Citation: Front. Neurosci. (2009) 3,1: 6-7. doi: 10.3389/ neuro.01.010.2009

Copyright $(\odot 2009$ Barceló. This is an open-access publication subject to an exclusive license agreement between the authors and the Frontiers Research Foundation, which permits unrestricted use, distribution, and reproduction in any medium, provided the original authors and source are credited.

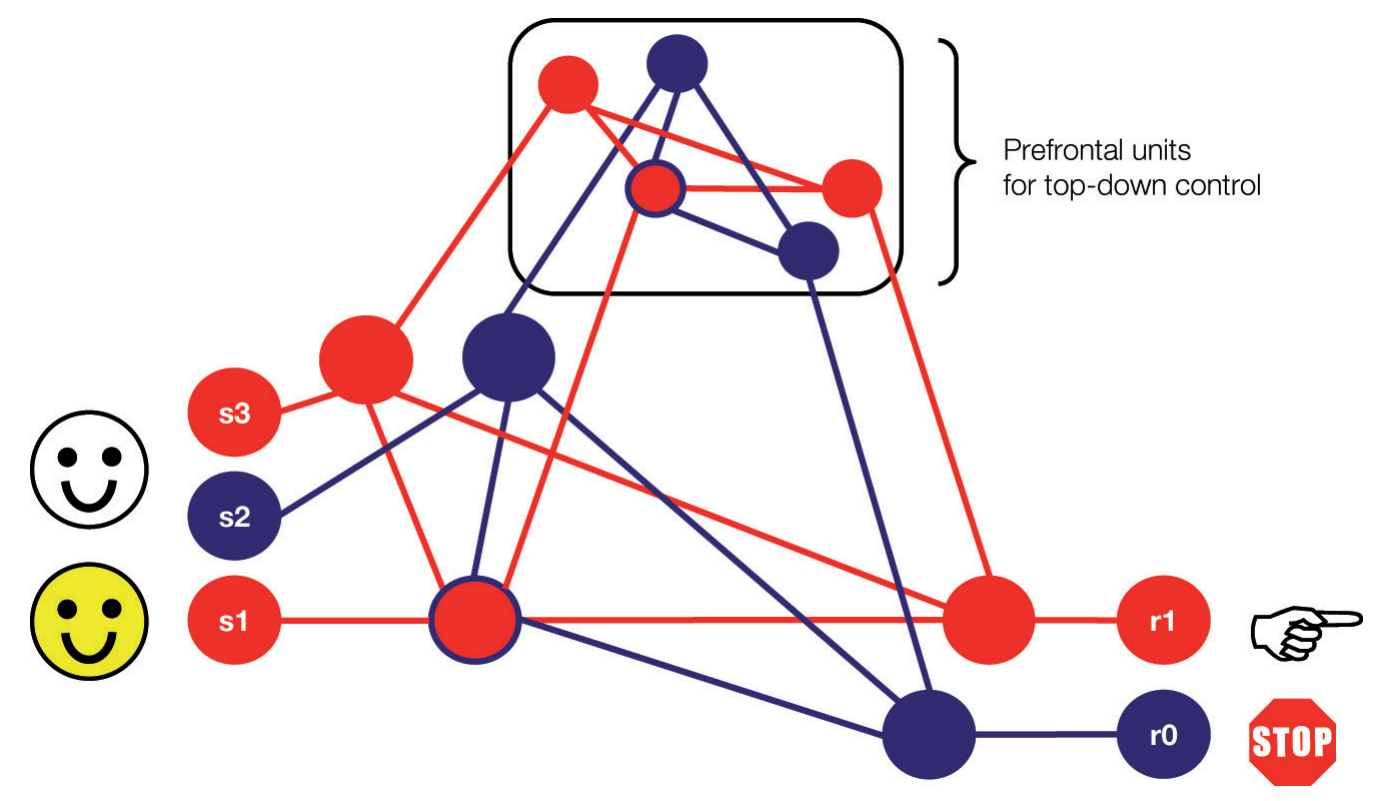

Figure 1 | Integrative model of prefrontal function (modified from Miller, 2000). Kiss et al.'s (2008) proposal could be framed within a general model of prefrontal inhibitory control by which distractor devaluation might result from attention-emotion interactions at various levels within a hierarchy of neural representations. The blue circles denote inhibitory tagging through Nogo pathways encompassing perceptual (s2), motor (r0), and prefrontal cortical units (i.e., anterior cingulate), as well as through lower-order intervening sensorimotor pathways involving subcortical and/or other posterior cortical structures. Here a color face designates the task-relevant pathways for perception ( $\mathrm{s} 1$ ) and action selection ( $\mathrm{r} 1$ ). Note that distractor and target faces share perceptual features (s3) that can induce interference among competing pathways at higher levels in the hierarchy. The probabilistic activation of Go and Nogo pathways determines the relative recruitment of prefrontal resources (Barceló and Knight, 2007), as well as the intensity of midfrontal negativities and related positive-going brain potentials (Nieuwenhuis et al., 2003). This schematic suggests that efficient response inhibition likely depends on the monitoring of response conflicts, resulting in working memory overload and transient loss of cognitive control induced by the distractors (Barceló et al., 2008). 